\title{
Next-to-next-to-leading order QCD analysis of the Gross-Llewellyn Smith sum rule and the higher twist effects
}

\author{
Jiří Chýla \\ Institute of Physics, Na Slovance 2, Prague 8, Czechoslavakia \\ and \\ Andrei L. Kataev \\ Theory Division, CERN, CH-1211 Geneva 23, Switzerland; \\ Institute for Nuclear Research, Moscow 117312, Russia円.
}

\begin{abstract}
We present the next-to-next-to-leading order QCD analysis of the Gross-Llewellyn Smith (GLS) sum rule in deep inelastic lepton-nucleon scattering, taking into account dimensiontwo, twist-four power correction. We discuss in detail the renormalization scheme dependence of the perturbative QCD approximations, propose a procedure for an approximate treatment of the quark mass threshold effects and compare the results of our analysis to the recent experimental data of the CCFR collaboration. From this comparison we extract the value of the strong coupling constant $\alpha_{s}^{n n l}\left(M_{Z}, \overline{\mathrm{MS}}\right)=0.115 \pm 0.001$ (stat) \pm 0.005 (syst) \pm 0.003 (twist) \pm 0.0005 (scheme). We stress the importance of an accurate measurement of the GLS sum rule and in particular of its $Q^{2}$ dependence.
\end{abstract}

CERN-TH.6604/92

August 1992

\footnotetext{
${ }^{1}$ Permanent address.
} 

1. In this paper we continue our investigation of the phenomenological aspects of the available next-to-next-to-leading order (NNLO) perturbative QCD approximations to measurable physical quantities, and in particular of their implications for a precise determination of the strong coupling constant $\alpha_{s}$ at different scales (see [1] for a recent detailed discussion of this point). In our previous publication [2] we have discussed in detail the NNLO QCD predictions for the familiar R-ratios in $\mathrm{e}^{+} \mathrm{e}^{-}$annihilation as well as for the $\tau$-lepton decay rate. The inclusion of the NNLO corrections calculated in [3] proved to be quite important, in particular for the latter quantity, as it significantly decreased the sensitivity of QCD predictions to the well-known renormalization scheme (RS) ambiguity [2]. This makes the $\tau$-lepton decay rate a suitable place for testing QCD (for more detailed discussions of this subject see refs. [4], [1]). Using the results of our work [2] the RS ambiguities were considered also in [5] for the case of the $\mathrm{e}^{+} \mathrm{e}^{-}$annihilation in the resonance region and in [6] for the $\tau$-lepton decay ratio.

Besides the above-mentioned two quantities, the NNLO calculations are available also for some of the deep-inelastic scattering sum rules [7, 8]. The corresponding next-to-leading order (NLO) calculations can be found in [9, 10]. In this paper we concentrate on the Gross-Llewellyn Smith (GLS) sum rule and after discussion of various kinds of theoretical ambiguities related to it, present a phenomenological analysis of recent experimental data from the CCFR Collaboration [1].

2. The quantity of interest in our case is the non-trivial part $\Delta$ of the GLS sum rule

$$
G L S=\frac{1}{2} \int_{0}^{1} d x F_{3}^{\bar{\nu} p+\nu p}\left(x, Q^{2}\right)
$$

defined as

$$
\Delta=(3-G L S) / 3
$$

As $\Delta=\Delta\left(Q^{2}\right)$ depends, through the $Q^{2}$ dependence of the structure function $F_{3}$ itself, on $Q^{2}$, it would be very useful to have this quantity measured in a broad range of $Q^{2}$ values. Unfortunately the actual situation with the GLS sum rule is more complicated, for experimental as well as theoretical reasons, and its analysis burdened with several problems related to the treatment of the low $x$ region. The point is that although $F_{3}^{\nu p}, F_{3}^{\bar{\nu} p}$ are functions of $x$ and $Q^{2}$ only, the integral in (11) cannot in practice be evaluated from experimental data at any finite primary energy. Indeed, the recent experimental analysis of the CCFR collaboration [1] gives

$$
\Delta_{\text {exp }}\left(\left\langle Q^{2}\right\rangle=3 \mathrm{GeV}^{2}\right)=0.167 \pm 0.006(\text { stat }) \pm 0.026 \text { (systematic) }
$$


At fixed $Q^{2}$ there is always a minimal value of accessible $x$, determined by the requirement that the total hadronic energy $W$, related to $x$ and $Q^{2}$ by

$$
W^{2}=Q^{2} \frac{1-x}{x}+m_{p}^{2}
$$

does not exceed $S$, the total lepton-proton centre of mass energy: $x_{\min }=Q^{2} /\left(S+Q^{2}-m_{p}^{2}\right)$. Small values of $x$ thus require small values of the ratio $Q^{2} / S$ and if we want to avoid large uncertainties due to extrapolation of experimental data to the low $x$ region, we are forced to include in the experimental determination of (1) a broad range of $Q^{2}$ values, down to a few $\mathrm{GeV}^{2}$. In this region we encounter the following theoretical complications:

- The quark mass thresholds. The integration over $x$ in (1) means that the corresponding values of $W$ span the whole region $S>W^{2}>m_{p}^{2}$. As all the NNLO calculations have been performed for massless quarks, we face the question of the appropriate treatment of quark mass thresholds. As for a given $Q^{2}$ the quantity determining the effective number of massless quarks to be used in evaluating (1) is $W$, we plot in Fig.1 as functions of $Q^{2}$ the relative contributions $w^{i}\left(Q^{2}\right)$ to the GLS sum rule $(i=3,4,5)$ of the three intervals

$$
\begin{gathered}
0<x<x_{1}, \quad x_{1}<x<x_{2}, \quad x_{2}<x<1 \\
x_{i}=\frac{Q^{2}}{Q^{2}+W_{i}^{2}-m_{p}^{2}}, \quad i=1,2, \quad W_{1}^{2}=4 m_{b}^{2}, W_{2}^{2}=4 m_{c}^{2}
\end{gathered}
$$

in which the effective $n_{f}$ equals 5,4 and 3 respectively $\left(m_{b}=4.5 \mathrm{GeV}, m_{c}=1.5 \mathrm{GeV}\right.$ are the $b$ and $c$-quark current on-shell masses [). The curves in Fig.1 correspond to three widely used sets of distribution functions: Duke-Owens set 1 [12], DFLM set 2 [13] and Morfin-Tung (fit $\mathrm{S}$ in $\overline{\mathrm{MS}}$ ) [14]. It is obvious that the fractions $w^{i}$ are only weakly sensitive to the specific choice of quark distribution functions. In evaluating the fractions $w^{i}$ we have used the parton model formula

$$
G L S=\frac{1}{2} \int_{0}^{1}\left(u_{v}(x)+d_{v}(x)\right) d x
$$

where $u_{v}(x)=u(x)-\bar{u}(x)$ and $d_{v}(x)=d(x)-\bar{d}(x)$ are the standard valence quark distribution functions. We see that the integral (1) is dominated by $n_{f}=3$ only for very low $Q^{2}$, while for $Q^{2}=3 \mathrm{GeV}^{2}$, which is the average value of $Q^{2}$ in the data of [11], $n_{f}=4$ gives the largest contribution, but with significant admixture of $n_{f}=3,5$. The sizeable $Q^{2}$ dependence of the fractions $w^{i}\left(Q^{2}\right)$ comes predominantly from the explicit $Q^{2}$-dependence of the boundary value $x_{i}, i=1,2$ and not from the $Q^{2}$-dependence of the distribution functions $u_{v}\left(x, Q^{2}\right), d_{v}\left(x, Q^{2}\right)$. Clearly, the only entirely consistent way of incorporating the flavour threshold effects would be to carry out all the calculations

\footnotetext{
${ }^{2}$ For $m_{c}=1.35 \mathrm{GeV}$, used in the CCFR analysis, our results change insignificantly.
} 
up to the NNLO for massive quarks. As this is practically impossible to do, we shall later formulate a parton model based procedure which we believe should reasonably approximate the effects of quark mass thresholds.

- Renormalization scheme dependence. For $Q^{2}$ in the region of a few $\mathrm{GeV}^{2}$ the RS ambiguities are expected to be phenomenologically important, as was the case in [2]. Moreover, for the GLS sum rule the RS dependence in the low $Q^{2}$ region turns out to depend sensitively on the value of $n_{f}$.

- Contribution of higher twists. Contrary to the case of the $\tau$-lepton decay, which is also characterized by a rather low value of the natural scale $Q^{2}$, the results [15, 16] give concrete estimates of the dimension-two, twist-four contribution (for recent theoretical discussions of the dimension-two contributions to the $\mathrm{e}^{+} \mathrm{e}^{-}$annihilation and the $\tau$-lepton decay R-ratios see [17, 1]). The estimates [16] suggest that the twist-four contribution is quite sizeable and has therefore to be taken into account.

3. The estimate of the corresponding twist-four contribution to the GLS sum rule, performed in [16] using QCD sum rules formalism, implies for our quantity $\Delta$

$$
\Delta_{\text {twist }-4}=\frac{8}{27} \frac{\left\langle\left\langle O^{S}\right\rangle\right\rangle}{Q^{2}}, \quad\left\langle\left\langle O^{S}\right\rangle\right\rangle=0.33 \mathrm{GeV}^{2}
$$

Although the typical QCD sum rules accuracy is about $30 \%$, we prefer to be conservative and threfore put a 50\% error bar on the above estimate. These errors are consistent with the estimate of the higher twist contribution obtained in [16] by two other methods, namely vector dominance approximation and the non-relativistic quark model. In [16] it was also shown that the results of the latter method indicate the problems in the related bag model calculations of [18, which gave negligibly small values of the twist-four contribution.

For $Q^{2}=3 \mathrm{GeV}^{2}$ we thus use

$$
\Delta_{\text {twist-4 }}=0.032 \pm 0.016
$$

Its central value is about $1 / 3$ of the leading perturbative correction in the $\overline{\mathrm{MS}} \mathrm{RS}$. The best way to detect the presence of the higher twist contributions would be to study the $Q^{2}$ evolution of the GLS sum rules. Experimentally this is, however, difficult to do and all data available so far therefore correspond to the averages over rather broad intervals of $Q^{2}$.

4. The perturbative part of $\Delta\left(Q^{2}\right)$ can be expanded in the renormalized couplant (we adopt the notation of [19) $a(\mathrm{RS})=\alpha_{s}(\mathrm{RS}) / \pi$

$$
\Delta_{\text {pert }}\left(Q^{2}\right)=a(\mathrm{RS})\left(1+r_{1}\left(Q^{2}, \mathrm{RS}\right) a(\mathrm{RS})+r_{2}\left(Q^{2}, \mathrm{RS}\right) a^{2}(\mathrm{RS})+\cdots\right)
$$

defined in a particular RS. As indicated in (2) both the couplant $a$ and the coefficients $r_{k}$ do depend on the chosen RS. For the discussion of the RS dependence of physical quantities 
like (2), the RS may be uniquely defined by the set $\left\{a, c_{k} ; k \geq 2\right\}$ where $c_{k}$ are related to the coefficients of the QCD $\beta$-function. Let us write the couplant $a(\mathrm{RS})$ as a function of the renormalization scale variable $\mu$,

$$
\frac{d a(\mu, R C)}{d \ln \mu}=\beta(a)=-b a^{2}\left(1+c a+c_{2} a^{2}+\cdots\right)
$$

where $b=\left(33-2 n_{f}\right) / 6, c=\left(153-19 n_{f}\right) /\left(66-4 n_{f}\right)$ are the RG invariants while $c_{i}, i>2$ are free parameters defining the so-called renormalization convention (RC). At the NNLO we have two free parameters labelling our RS: $c_{2}$ and either $a$ itself or $\mu$, related to it in (9). The consistency conditions lead to the following explicit relations [19]

$$
\begin{gathered}
r_{1}=b \ln (\mu / \bar{\Lambda})-\rho \\
r_{2}=\rho_{2}-c_{2}+\left(r_{1}+c / 2\right)^{2}
\end{gathered}
$$

where

$$
b \ln (\mu / \bar{\Lambda})=\frac{1}{a}+c \ln \left(\frac{c a}{\sqrt{1+c a+c_{2} a^{2}}}\right)+f\left(a, c_{2}\right)
$$

and

$$
\begin{aligned}
f\left(a, c_{2}\right) & =\frac{2 c_{2}-c^{2}}{d}\left(\arctan \frac{2 c_{2} a+c}{d}-\arctan \frac{c}{d}\right), \quad d=\sqrt{4 c_{2}-c^{2}}, 4 c_{2}>c^{2} \\
& =\frac{2 c_{2}-c^{2}}{2 d}\left(\ln \left|\frac{2 c_{2} a+c-d}{2 c_{2} a+c+d}\right|-\ln \left|\frac{c-d}{c+d}\right|\right), \quad d=\sqrt{c^{2}-4 c_{2}}, 4 c_{2}<c^{2}
\end{aligned}
$$

In $(10,11) \rho=\rho\left(\sqrt{Q^{2}} / \bar{\Lambda}\right)$ and $\rho_{2}$ are RG invariants [19] and $\bar{\Lambda}$ is defined as

$$
\bar{\Lambda}=\Lambda(2 c / b)^{-c / b}
$$

where $\Lambda$, which is held fixed, is the conventional definition of the QCD $\Lambda$-parameter. Combining $(10,12)$ we find

$$
r_{1}=\frac{1}{a}+c \ln \left(\frac{c a}{\sqrt{1+c a+c_{2} a^{2}}}\right)+f\left(a, c_{2}\right)-\rho
$$

and putting all together we obtain $\Delta_{\text {pert }}^{n n l}$ as a function of $a, c_{2}, \rho$ and $\rho_{2}$. Note that the energy dependence of $\Delta_{\text {pert }}^{n n l}$ enters entirely through the RG invariant $\rho$ which can be written as

$$
\rho=b \ln \left(\sqrt{Q^{2}} / \bar{\Lambda}_{\overline{\mathrm{MS}}}\right)-r_{1}\left(\mu=\sqrt{Q^{2}}, \overline{\mathrm{MS}}\right)
$$

where we take for the referential RS the $\overline{\mathrm{MS}}$ one.

The RS dependence of $\Delta_{p e r t}^{n n l}\left(a, c_{2} ; \rho, \rho_{2}\right)$ can therefore be represented by a two-dimensional surface in three dimensions. In this picture each point on such a surface represents uniquely one RS. Recall that at the NLO $\Delta_{\text {pert }}^{n l}$ was given simply as [19]

$$
\Delta_{p e r t}^{n l}=a(2+c a \ln (c a /(1+c a))-\rho a)
$$


and the corresponding curve was close to a parabola. At the NNLO the surface representing $\Delta^{n n l}$ depends non-trivially on the mutual relation of the two RG invariants $\rho$ and $\rho_{2}$, and in particular on the sign of the latter one. For the $\mathrm{e}^{+} \mathrm{e}^{-}$annihilation and $\tau$ decay R-ratios, $\rho_{2}<0$ for all $n_{f} \geq 3$ and so only this case was discussed in [2]. For (1) the situation is more complicated as now both cases $\rho_{2}<c^{2} / 4$ and $\rho_{2}>c^{2} / 4$ are physically relevant.

In [8] the NNLO calculations of (1) were carried out in the $\overline{\mathrm{MS}} \mathrm{RS}$ with the result

$$
r_{2}=41.441-8.02 n_{f}+0.177 n_{f}^{2}
$$

which coupled with the earlier known formulae for $r_{1}$ [10], and $c_{2}$ [20] in the same $\overline{\mathrm{MS}} \mathrm{RS}$

$$
\begin{gathered}
r_{1}=55 / 12-n_{f} / 3 \\
c_{2}=\frac{77139-15099 n_{f}+325 n_{f}^{2}}{9504-576 n_{f}}
\end{gathered}
$$

yields an explicit dependence of $\rho_{2}\left(n_{f}\right)$ ङ. In particular

$$
\rho_{2}(3)=3.438, \quad \rho_{2}(4)=-0.928, \quad \rho_{2}(5)=-5.351
$$

To get a quantitative idea of the shape of $\Delta_{p e r t}^{n n l}$ as a function of $a$ and $c_{2}$, for given $\rho$ and $\rho_{2}$, we look for the stationary points with respect to the variation of $a$, given by the solutions to the equation

$$
\frac{d \Delta_{p e r t}^{n n l}}{d a}=0
$$

In [2] we discussed in detail the situation for $\rho_{2}<0$; here we briefly sketch what it looks like for $\rho_{2}>0$. In this case the solutions of (23) make up a curve in the plane $a, c_{2}$ and lie in the quadrant $a>0, c_{2}>0$. For sufficiently large $\rho$, there lies along each such curve a saddle point defining the PMS choice of the RS. For large values of the couplant, relevant for the IR region, the functional form of the curves, i.e. $a\left(c_{2}, \rho, \rho_{2}\right)$, can be expressed analytically. Expanding (16) in powers of $1 / a$ and keeping only the non-vanishing terms we get, using (10, 11)

$$
\Delta_{\text {pert }}^{n n l}=\left(\rho_{2}-c_{2}+(\gamma-\rho)^{2}\right) a^{3}+\left(\gamma-\frac{c}{2}-\rho\right) a^{2}+a+2(\gamma-\rho) \kappa+O(1 / a)
$$

where $\kappa=1 /\left(3 c_{2}\right)$ and

$$
\gamma=c \ln \left(c \sqrt{c_{2}}\right)+\frac{2 c_{2}-c^{2}}{\sqrt{4 c_{2}-c^{2}}}\left(\frac{\pi}{2}-\arctan \frac{c}{\sqrt{4 c_{2}-c^{2}}}\right)+\frac{c}{2}
$$

\footnotetext{
${ }^{3}$ Here we included in $\rho_{2}$ the contributions of the light-by-light-type graphs contributing to $r_{2}$. For the phenomenologically interesting case $\rho>0$ the separation of these contributions in accordance with the proposal of [21] has an entirely negligible effect on the results of this analysis.
} 
The equation (23) has two simple physical solutions

$$
\begin{aligned}
& a_{1}=\frac{\rho-\gamma+c / 2+\sqrt{3\left(c_{2}-\rho_{2}\right)+(\rho+c / 2-\gamma)^{2}-3(\rho-\gamma)^{2}}}{3\left(\rho-\gamma+\sqrt{c_{2}-\rho_{2}}\right)\left(\rho-\gamma-\sqrt{c_{2}-\rho_{2}}\right)} \\
& a_{2}=\frac{\rho-\gamma+c / 2-\sqrt{3\left(c_{2}-\rho_{2}\right)+(\rho+c / 2-\gamma)^{2}-3(\rho-\gamma)^{2}}}{3\left(\rho-\gamma+\sqrt{c_{2}-\rho_{2}}\right)\left(\rho-\gamma-\sqrt{c_{2}-\rho_{2}}\right)}
\end{aligned}
$$

which coincide for $c_{2}^{0}$ given as the solution to the equation

$$
3\left(c_{2}-\rho_{2}\right)+(\rho+c / 2-\gamma)^{2}-3(\rho-\gamma)^{2}=0
$$

At that point

$$
a_{1}=a_{2}=\frac{1}{\rho-\gamma+c / 2} \Rightarrow \infty \quad \text { as } \rho \Rightarrow(\gamma-c / 2)
$$

For $\rho<(\gamma-c / 2)$ only $a_{2}$ stays positive and is thus physically acceptable. As we decrease $\rho$ even further the curves move upwards and simultaneously shrink to a point at some $\overline{c_{2}}$. The lower bound on $\rho$ follows again from the requirement $a_{2}>0$ which means

$$
\rho>h\left(c_{2}\right)=\gamma\left(c_{2}\right)-\sqrt{c_{2}-\rho_{2}}
$$

The minimum of the function $h\left(c_{2}\right)$ lies at $\overline{c_{2}}$ given as the solution of the equation

$$
-c \sqrt{4 c_{2}-c^{2}}+2 c_{2}\left(\pi-2 \arctan \frac{c}{\sqrt{4 c_{2}-c^{2}}}\right)-\frac{\sqrt{4 c_{2}-c^{2}}\left(4 c_{2}-c^{2}\right)}{2 \sqrt{c_{2}-\rho_{2}}}=0
$$

Substituting the solution of (31) into (30) we get the lower bound $\rho_{\min }^{P M S}$ as a function of $\rho_{2}$. For $\rho<\rho_{\min }^{P M S}, \Delta_{\text {pert }}^{n n l}$ is again a monotonous function of the couplant for any $c_{2}$. Solving (31) for $n_{f}=3$ we obtain

$$
\rho_{\min }^{P M S}=0.863, \overline{c_{2}}=\lim _{\rho \rightarrow \rho_{\min }} c_{2}^{P M S}(\rho)=12.29
$$

It should be stressed that the basic idea of the method of the "effective charges"(EC) [22] (or the scheme-invariant perturbation theory [23]) should be considered more carefully when we go from the NLO to the NNLO. Indeed the condition

$$
r^{E C}=a^{E C}
$$

implies at the NNLO merely

$$
r_{1}+r_{2} a=0
$$

which has an infinite number of solutions corresponding to the intersection of the surface $\Delta_{\text {pert }}^{n n l}\left(a, c_{2}\right)$ with the plane $\Delta=a$. There are two, one or no intersections for any given $c_{2}$ , depending on the values of $\rho$ and $\rho_{2}$. At first glance there is no obvious reason to single out one of them. However, requiring $r_{1}, r_{2}$ to vanish separately (as is assumed in 22]) fixes 
$c_{2}=\rho_{2}+(c / 2)$ as well as $a$. In the following this is what we call the EC result. The equation determining the value $\rho_{\min }^{E C}$ at which $a_{E C}^{n n l} \rightarrow \infty$ reads

$$
\rho_{\min }^{E C}=c \ln \frac{c}{\sqrt{\rho_{2}+c^{2} / 4}}+\frac{4 \rho_{2}-c^{2}}{4 \sqrt{\rho_{2}}}\left(\frac{\pi}{2}-\arctan \frac{c}{2 \sqrt{\rho_{2}}}\right)
$$

and has for $n_{f}=3$ the solution

$$
\rho_{\min }^{E C}(3)=1.35
$$

At the NNLO and for $n_{f}=4$ the IR fixed points of the PMS and EC NNLO approximants are at

$$
a^{* P M S}=1.12, \quad c_{2}^{* P M S}=-2.18, \quad a^{* E C}=5.17 \quad c_{2}^{* E C}=-0.335
$$

while for $n_{f}=5$

$$
a^{* P M S}=0.371, \quad c_{2}^{* P M S}=-10.62, \quad a^{* E C}=0.594 \quad c_{2}^{* E C}=-4.95
$$

We shall not advocate here any of the popular choices of the RS (PMS, EC or $\overline{\mathrm{MS}}$ ) but shall take the difference between the PMS/EC and the $\overline{\mathrm{MS}}$ results as a measure of the RSdependence (in the phenomenologically relevant region of $\rho$ the EC results are practically indistinguishable from those of the PMS). As we shall see, typical values of $\rho$ appropriate to the CCFR data [1] lie in the region $\rho \in(2,6)$, while $\rho$ up to 25 might be of interest at currently accessible values of $Q^{2}$.

5. The main difference between the $\mathrm{RS}$ dependences for $n_{f}=3,4,5$ lies in the IR region, which is most of all influenced by the fact that $\rho_{2}<0$ for $n_{f}=4,5$ and $\rho_{2}>0$ for $n_{f}=3$ (see (22)) implying no IR stability at the NNLO for $n_{f}=3$ even in the PMS and EC approaches. The question to what extent is the IR stability, observed for $n_{f}=4,5$ in certain schemes, is of physical relevance or merely an artefact of finite order calculations and/or the choice of the RS, is difficult to answer on the basis of the available perturbative calculations themselves. Nevertheless, if it should really be the case, then a similar behaviour would certainly have to be observed for $n_{f}=3$ as well. As this is not the case the IR stability of the NNLO PMS and EC approximants for $n_{f}=4,5$, characterized by (36),(37), has probably little physical relevance. Moreover, due to a rather small magnitude of $\rho_{2}, \Delta_{\text {pert }}^{n n l}$ has IR zeros of the PMS and EC approximants at so large values of the couplant that the NNLO approximations can hardly be trusted. In the following we shall discuss only the region of positive $\rho$ where the problem of asymptotic explosion does not arise.

In Fig. 2 we plot the dependence $\Delta_{\text {pert }}^{n n l}(\rho)$ on $\rho$ for all three RS: PMS/EC and $\overline{\mathrm{MS}}$ and for $n_{f}=3,4,5$. For PMS/EC results we observe sizeable $n_{f}$ dependence in particular in the small $\rho$ region. This difference is significant up to $\rho \sim 5$. On the other hand the $\overline{\mathrm{MS}}$ results are nearly $n_{f}$ independent!. This somewhat surprising effect is a result of nontrivial partial compensation between significant $n_{f}$-dependences of the coefficients $r_{k}, k=1,2$, as given in 
(20,19), and the couplant $a$ in the $\overline{\mathrm{MS}} \mathrm{RS}$, induced by the $n_{f}$-dependence of the $\beta$-function coefficients $b_{k}$.

The same facts, but viewed differently, are presented in Fig.3 where we plot the RS dependence, as measured by the difference between the PMS/EC and $\overline{\mathrm{MS}}$ results, for $n_{f}=$ $3,4,5$. The PMS and EC approaches are practically indistinguishable in our plots. At the NNLO and for fixed $\rho$ the RS dependence diminishes as $n_{f}$ grows, while at the NLO it remains about the same. The importance of the NNLO corrections with respect to the NLO ones can be assessed from Fig.4, where we plot the comparison between NLO and NNLO as a function of $\rho$ for all three values if $n_{f}$ and for both PMS/EC and $\overline{\mathrm{MS}} \mathrm{RS}$. We see that

- in the $\overline{\mathrm{MS}} \mathrm{RS}$ we find $\Delta_{\text {pert }}^{n l}(\rho)<\Delta_{\text {pert }}^{n n l}(\rho)$ for all values of $\rho$, the difference $\Delta_{\text {pert }}^{n n l}-\Delta_{\text {pert }}^{n l}$ being a decreasing function of $n_{f}$;

- in the PMS/EC approaches the difference $\Delta_{\text {pert }}^{n l}-\Delta_{\text {pert }}^{n n l}$ is positive for $n_{f}=5$, close to zero for $n_{f}=4$ and negative for $n_{f}=3$. Moreover this difference is sizeable and thus of phenomenological relevance. For the consequences of these two observations see further discussion;

- in the phenomenologically important region of $Q^{2}$ the incorporation of the NNLO corrections into the analysis decreases the difference between the PMS/EC and $\overline{\mathrm{MS}}$ results.

The curves shown in Figs. 2,3,4 can be used for straightforward determination of the QCD parameter $\Lambda_{\overline{\mathrm{MS}}}$ from experimental data on $\Delta$. For easy use we have fitted them by an analytical expression of the form

$$
\Delta_{\text {pert }}^{(i)}(\rho)=\sum_{j=0}^{5} \frac{r_{j}^{(i)}}{\rho^{j}}
$$

in the wide interval $\rho \in(2,26)$, with $i$ labelling one of the 18 combinations of the order (NLO or NNLO), RS (PMS, EC or $\overline{\mathrm{MS}}$ ) and $n_{f}(3,4,5)$. The values of all the parameters $r_{j}^{(i)}$ are given in Table 1 . The extraction of $\Lambda_{\overline{\mathrm{MS}}}$ from a given experimental value $\Delta_{\text {exp }}$ proceeds in two steps: first one solves the equation $\Delta_{\text {pert }}^{(i)}(\rho)=\Delta_{\text {exp }}$ and then uses the solution $\rho_{\text {exp }}^{(i)}$ in the formula

$$
\Lambda \frac{(i)}{\mathrm{MS}}=Q \exp \left[-\left(\frac{\left.r_{1}(\overline{\mathrm{MS}})+\rho_{e x p}^{(i)}\right)}{b}\right)\right]\left(\frac{2 c}{b}\right)^{c / b}
$$

The analytical parametrization of the dependences $\Delta_{\text {pert }}^{(i)}(\rho)$ is useful also for the determination of the error $\sigma_{\Lambda}$ of the extracted $\Lambda_{\overline{\mathrm{MS}}}$ from the error $\sigma_{\Delta}$ on $\Delta_{\text {exp }}$ :

$$
\frac{\sigma_{\Lambda}}{\Lambda_{\overline{\mathrm{MS}}}}=\frac{\sigma_{\Delta}}{b|d \Delta(\rho) / d \rho|}
$$


The relation (39) holds for massless quarks and thus makes good sense only provided $n_{f}$ is well defined and fixed. As in practice this is usually not the case, we have developed a procedure which takes into account at least in an approximate way the quark mass thresholds. For all four combinations of the order (NLO and NNLO) and RS (EC/PMS and $\overline{\mathrm{MS}}$, EC and PMS being indistinguishable for our purposes), it consists of the following steps:

1. From the information on valence distribution functions we determine, for a given $Q^{2}$, the fractions $w^{i}\left(Q^{2}\right)$ defining the relative importance of the contribution of $n_{f}=3,4,5$ to (1). We do this by taking the average of results corresponding to the three mentioned sets of quark distribution functions. For $Q^{2}=3 \mathrm{GeV}^{2}$ we find: $w^{1}=0.20, w^{2}=$ $0.51, w^{3}=0.29$.

2. From (39) $\Lambda \frac{\left(n_{f}\right)}{\mathrm{MS}}$ appropriate for $n_{f}$ massless quarks is determined.

3. Using the formulae from 24 these values are then translated to $\Lambda \frac{(4)}{\mathrm{MS}}\left(n_{f}\right)$ where the $\operatorname{argument} n_{f}$ keeps track of the source of this resulting $\Lambda \frac{(4)}{\mathrm{MS}}$.

4. The average value $\tilde{\Lambda}_{\frac{(4)}{\mathrm{MS}}}=\sum_{i=3}^{5} w^{i} \Lambda \frac{(4)}{\mathrm{MS}}(i)$ similarly for errors) is evaluated.

5. Using the average value $\tilde{\Lambda}_{\frac{(4)}{\mathrm{MS}}}$ we go back and calculate the corresponding values of $\Lambda \frac{\left(n_{f}\right)}{\mathrm{MS}}$.

6. From these values of $\Lambda \frac{\left(n_{f}\right)}{\mathrm{MS}}$ we calculate, according to (17), the corresponding $\rho\left(n_{f}\right)$ and then finally evaluate the averages $\bar{\Delta}_{\text {pert }}=\sum_{i=3}^{5} w^{i} \Delta_{\text {pert }}\left(\rho_{i}\right)$.

For this procedure to be self-consistent we should come at the end of step 6 close to the experimental value of $\Delta_{\exp }$ 3. If the data do not correspond to a fixed value of $Q^{2}$ the whole procedure should be folded with the known $Q^{2}$ dependence of the data. As this is difficult to do we have carried out the above procedure for fixed $Q^{2}=3 \mathrm{GeV}^{2}$, equal to the average value of $Q^{2}$ in the data of [11]. We have included the higher twist contribution as given in (7) t but carried out the whole analysis also for the case of no higher twists at all. In Table 2 we present in detail the results of our procedure for the case of the central value of $\Delta_{\text {twist-4 }}=0.032$. Notice that the averages in the last column are indeed quite close to $\Delta_{\text {exp }}-0.032=0.135$. Similar tables have been constructed for the upper (0.048) as well as lower (0.016) estimates of twist-4 contributions and also for the case of no twist-4 contribution at all. In these latter cases only the extracted values of $\Lambda \frac{(4)}{\mathrm{MS}}$ are presented in Table 3. From Tables 2 and 3 we conclude that:

\footnotetext{
${ }^{4}$ We neglected in our analysis the kinematical power corrections and the twist-six corrections since it was shown in ref. 16] that they are significantly smaller than the central value of the twist-four contributions (7). It might be interesting to take these effects into account in order to compare their value with the assumed by us $50 \%$ error bars of the twist-four contibutions.
} 
- the inclusion of the higher twists is quite important as it lowers the central value of $\Lambda_{\overline{\mathrm{MS}}}$ by about $100 \mathrm{MeV}$;

- in the $\overline{\mathrm{MS}}$ as well as PMS/EC approaches $\Lambda \frac{(4)}{\mathrm{MS}}\left(\mathrm{NNLO}, n_{f}=3\right)<\Lambda \frac{(4)}{\mathrm{MS}}\left(\mathrm{NLO}, n_{f}=3\right)$ while in the PMS/EC approach they are almost the same (smaller) for $n_{f}=4\left(n_{f}=5\right)$. This is a consequence of the first two observations of Sec.5, namely, that $\Delta_{\text {pert }}^{n l}<\Delta_{\text {pert }}^{n n l}$ in the $\overline{\mathrm{MS}} \mathrm{RS}$ and for PMS/EC with $n_{f}=3$, while in the latter case for $n_{f}=4\left(n_{f}=5\right)$ we have $\Delta_{\text {pert }}^{n l} \approx \Delta_{\text {pert }}^{n n l}\left(\Delta_{\text {pert }}^{n l}>\Delta_{\text {pert }}^{n n l}\right)$ (see Fig.4);

- the inclusion of the NNLO corrections reduces substantially the RS dependence as measured by the difference between the results for PMS/EC and $\overline{\mathrm{MS}}$ approaches while its influence on $\Lambda_{\overline{\mathrm{MS}}}$ is smaller than the effects of the twist-four corrections; due to

- the errors of the extracted $\Lambda \frac{(4)}{\mathrm{MS}}$ values are dominated on one side by the systematical experimental uncertainties and on the other by uncertainty in the higher twist contribution. The reduction of the systematical experimental errors (which are the biggest single source of errors and make about half of the total error), to the level of statistical ones would make the GLS sum rules a very good place for testing perturbative QCD provided a more accurate estimate of the higher twist were to be available. But even taking into account the combined effect of all discussed errors the accuracy of the extracted $\Lambda_{\overline{\mathrm{MS}}}$ is not bad;

- the results of the averaging procedure are not far from those which correspond to fixed $n_{f}=4$. This is due to the fact that for $Q^{2}=3 \mathrm{GeV}^{2} n_{f}=4$ is dominant while $n_{f}=3,5$ contribute with comparable strength, thus roughly balancing each other. For other $Q^{2}$ the situation may be quite different.

6. Let us now summarize our results. At the NLO and in the $\overline{\mathrm{MS}} \mathrm{RS}$ we find

$$
\Lambda \frac{(4)}{\mathrm{MS}}=317 \pm 23(\text { stat }) \pm 99(\text { syst }) \pm 62 \text { (twist) } \mathrm{MeV}
$$

while the NLO PMC/EC result reads

$$
\Lambda \frac{(4)}{\mathrm{MS}}=241 \pm 14(\text { stat }) \pm 60(\text { syst }) \pm 40 \text { (twist) } \mathrm{MeV}
$$

where the third error, due to higher twist contributions corresponds to the limits given in (7). Notice that at the NLO the value of $\Lambda \frac{(4)}{\mathrm{MS}}$ extracted within the PMS/EC approach is smaller than the one obtained in the $\overline{\mathrm{MS}}$ analysis. A similar situation occurs in the NLO analysis of the BCDMS data on $\mathrm{F}_{2}\left(x, Q^{2}\right)$ without higher twist contributions [25] and in the NLO analysis of the BCDMS and SLAC data with higher twist contributions [26]. Our analysis differs from that of [26] and other similar ones in the way higher twists are treated. While we use concrete estimates for twist-four contributions (though with sizeable errors), 
previously they were simply parametrized by the corresponding free parameters which were fitted together with pure perturbative expressions from experimental data.

At the NNLO the difference between the $\overline{\mathrm{MS}}$ and the PMS/EC results becomes considerably smaller:

$$
\begin{aligned}
\overline{\mathrm{MS}}: & \Lambda_{\overline{\mathrm{MS}}}^{(4)}=265 \pm 18(\text { stat }) \pm 80(\text { syst }) \pm 50(\text { twist }) \mathrm{MeV} \\
\mathrm{PMS} / \mathrm{EC}: & \Lambda_{\overline{\mathrm{MS}}}^{(4)}=249 \pm 16(\text { stat }) \pm 70(\text { syst }) \pm 45(\text { twist }) \mathrm{MeV}
\end{aligned}
$$

For a careful reader we add the following comment concerning the different relation between the errors at the NLO and the NNLO in PMS/EC and $\overline{\mathrm{MS}} \mathrm{RS}$. As follows from Figs. 2,3,4, in the PMS/EC approaches the central NNLO value of $\Lambda \frac{(4)}{\mathrm{MS}}$ is slightly larger than the NLO one. Furthermore, for $n_{f}=4$ the derivative of the PMS/EC approximant to $\Delta(\rho)$ is somewhat bigger at the NLO than at the NNLO. This leads (see (40)) to slightly lower error $\sigma_{\Lambda}$ at the NLO. The combination of these two small effects results in a slight increase of both theoretical and experimental uncertainties of $\Lambda \frac{(4)}{\mathrm{MS}}$ at the NNLO in PMS/EC approach compared to the corresponding NLO analysis (compare (43) with (41)).

Combining the $\overline{\mathrm{MS}}$ and PMS/EC results we finally get

$$
\begin{aligned}
\Lambda \frac{(4)}{\mathrm{MS}}(\mathrm{NLO}) & =279 \pm 19(\text { stat }) \pm 80(\text { syst }) \pm 50(\text { twist }) \pm 38(\text { scheme }) \mathrm{MeV} \\
\Lambda \frac{(4)}{\mathrm{MS}}(\mathrm{NNLO}) & =257 \pm 17(\text { stat }) \pm 75(\text { syst }) \pm 47(\text { twist }) \pm 8(\text { scheme }) \mathrm{MeV}
\end{aligned}
$$

Notice that with respect to the NLO results the NNLO statistical, systematical and higher twist uncertainties are slightly smaller, while the error due to the RS-dependence is reduced significantly. This is the main effect of including the NNLO corrections in the analysis of GLS sum rule. Our results are in very good agreement with those of the detailed NLO analysis [27] (carried out in the $\overline{\mathrm{MS}} \mathrm{RS}$ and including the phenomenological parametrization of the higher-twists effects) of combined BCDMS and SLAC data on $\mathrm{F}_{2}\left(x, Q^{2}\right)$ structure function:

$$
\Lambda \frac{(4)}{\mathrm{MS}}=263 \pm 42(\exp ) \mathrm{MeV}
$$

As, however, our analysis suggests the importance of including the NNLO corrections, it would be very desirable to investigate the influence of the yet uncalculated NNLO corrections to $\mathrm{F}_{2}\left(x, Q^{2}\right)$ on the result $(47)$ as well.

So far our analysis has been aimed primarily at the determination of $\Lambda \frac{(4)}{\mathrm{MS}}$ but it tells us also something about the role of the higher twist contributions to the GLS sum rule. Indeed, Table 1 suggests that if the twist-four contribution (7) is ignored or its magnitude is negligibly small (like in the bag model calculations [18]), the agreement between the results of our analysis and those of [27] worserns. This lends a posteriori support to the QCD sum

\footnotetext{
${ }^{5}$ The recent NLO $\overline{\mathrm{MS}}$ and scheme-invariant fits [26] of the BCDMS and SLAC data, including the higher twists, gave smaller values of $\Lambda_{\overline{\mathrm{MS}}}$. It would be interesting to understand the origin of these smaller values.
} 
rules estimate [16] of the higher twist effects. An independent and more accurate estimate of these higher twists, using for instance lattice methods (for a review of such calculations see [28]), would, however, be very welcome. This analysis might also shed the light on the role of other possible $1 / Q^{2}$-contributions, recently discussed, from a purely theoretical point of view, in [17, 1]. We intend to consider related problems in the future.

The above results on $\Lambda \frac{(4)}{\mathrm{MS}}$ imply for $\alpha_{s}$ at the scale $\sqrt{Q^{2}}=\sqrt{3} \mathrm{GeV}$ the following values

$$
\begin{aligned}
& \alpha_{s}^{n l}\left(\sqrt{Q^{2}}, \overline{\mathrm{MS}}\right)=0.318 \pm 0.010(\text { stat }) \pm 0.042 \text { (syst) } \pm 0.026 \text { (twist) } \pm 0.020(\text { scheme }) \\
& \left.\alpha_{s}^{n n l}\left(\sqrt{Q^{2}}, \overline{\mathrm{MS}}\right)=0.315 \pm 0.010(\text { stat }) \pm 0.044(\text { syst }) \pm 0.028(\text { twist }) \pm 0.005 \text { (scheme }\right)
\end{aligned}
$$

where in accordance with the discussions of Sec.2 we have taken $n_{f}=4$. The errors $\sigma_{\alpha_{s}}$ of the above results on $\alpha_{s}$ are related to the errors $\sigma_{\Lambda}$ of $(44),(45)$ as follows

$$
\sigma_{\alpha_{s}}=\frac{\sigma_{\Lambda}}{\Lambda} \frac{\pi b}{|d F / d a|}
$$

where $F$ is the r.h.s. of eq.(10).

Finally, to facilitate easy comparison with other determinations of $\alpha_{s}$ we have evolved it, using the fomulae of [24], and eqs.(48),(49), through the b-quark threshold up to $M_{Z}$ and obtained

$$
\begin{aligned}
\alpha_{s}^{n l}\left(M_{Z}, \overline{\mathrm{MS}}\right) & =0.116 \pm 0.001(\text { stat }) \pm 0.005(\text { syst }) \pm 0.003 \text { (twist }) \pm 0.002(\text { scheme }) \\
\alpha_{s}^{n n l}\left(M_{Z}, \overline{\mathrm{MS}}\right) & =0.115 \pm 0.001(\text { stat }) \pm 0.005(\text { syst }) \pm 0.003(\text { twist }) \pm 0.0005 \text { (scheme })
\end{aligned}
$$

These results can be compared with

$$
\alpha_{s}\left(M_{Z}, \overline{\mathrm{MS}}\right)=0.113 \pm 0.003(\exp ) \pm 0.004(\text { theor })
$$

obtained in the NLO analysis in the $\overline{\mathrm{MS}} \mathrm{RS}$ [27], where the standard two-loop inverse-log approximation for $\alpha_{s}$ was used. The theoretical error comes from the NLO estimates of the RS and factorization-scheme uncertainties [27] 9.

Our results also agree with the recent world average [1]

$$
\alpha_{s}\left(M_{Z}, \overline{\mathrm{MS}}\right)=0.112 \pm 0.007
$$

determined on the basis of the results of various deep-inelastic scattering experiments. The quoted error bars correspond to the combination of experimental and theoretical uncertainties with the dominant role of the latter ones. This average has in turn been used in the determination of the overall world average [1]

$$
\alpha_{s}\left(M_{Z}, \overline{\mathrm{MS}}\right)=0.118 \pm 0.007
$$

\footnotetext{
${ }^{6}$ We do not use this approximation but are solving (12) numerically to get $\alpha_{s}$ as a function of the scale $\mu=\sqrt{Q^{2}}$. Within this procedure, we got at the NLO 0.115 instead of the above mentioned result 0.113 . This theoretical difference lies within the total theoretical error of the result (53).
} 
with the typical error (mainly theoretical one). This world average sums up all available results on $\alpha_{s}$, including the relatively larger values coming from the analyses of the R-ratio in the $\mathrm{e}^{+} \mathrm{e}^{-}$annihilations into hadrons and of the total width of $Z^{0}$ decay into hadrons, both of which, however, have rather large experimental errors (see [2, 29, 30, 1]).

7. In this work we have presented the NNLO QCD analysis of the GLS sum rules using the new data of the CCFR Collaboration [11]. The inclusion of the NNLO corrections substantially improves the situation as far as the RS dependence is concerned and makes this quantity a potentially good place for testing the perturbative QCD. Our result

$$
\alpha_{s}^{n n l}\left(M_{Z}, \overline{\mathrm{MS}}\right)=0.115 \pm 0.001(\text { stat }) \pm 0.005 \text { (syst) } \pm 0.003(\text { twist }) \pm 0.0005 \text { (scheme) }
$$

is in good agreement with the current world average. However, three problems, two theoretical and the other experimental, stand, in the way of a more precise determination of $\alpha_{s}\left(M_{Z}\right)$ or $\Lambda_{\overline{\mathrm{MS}}}$ from this data. The first one concerns the treatment of flavour thresholds and we have suggested an approximate procedure to take this effect into account while still using the calculations for massless quarks. The second problem is connected with the necessity of getting more precise estimates of the theoretical errors of the higher twist terms. The third problem is related to the experimental errors, which are dominated by the systematical ones. These errors are currently larger then the theoretical errors of the higher twist terms and any attempt to reduce them would thus be very usefull. It would also be very interesting to have accurate data on (1) as a function of $Q^{2}$ in a broad range of $Q^{2}$ values. Their analysis would certainly lead to better understanding of the role of the higher twist contributions to deep inelastic scattering at low and moderate $Q^{2}$.

We intend to carry out an analysis similar to the one presented in this paper also for the Bjorken sum rule for polarized electroproduction structure functions, since the appropriate NNLO perturbative calculations [8] as well as power correction estimates [31], inspired by the analysis of [32], are available and the relevant experimental data will soon be obtained by CERN and SLAC groups.

\section{Acknowledgements}

We are gratefull to the organizers of the XXVII Rencontre de Moriond, where this work was started, and of the "QCD-20 Years Later" Conference, where our preliminary results were presented, for the invitations to Les Arcs, France and to Aachen, Germany. We wish to thank J.Blumlein for comments on error handling in extraction of $\Lambda_{\overline{\mathrm{MS}}}$. Special thanks are due to G. Altarelli for useful discussions of the results of our analysis. A. K. wishes to thank the members of the Theory Division of CERN for their kind hospitality. 


\section{References}

[1] G. Altarelli, Talk at the "QCD-20 Years Later" Conf. 9-13 June 1992, Aachen; to appear in the Proceedings; preprint CERN-TH.6623/92 (1992).

[2] J. Chýla, A. L. Kataev and S. A. Larin, Phys.Lett. B267 (1991) 269.

[3] S. G. Gorishny, A. L. Kataev and S. A. Larin, in "Standard Model and Beyond" Proc. Int. Workshop CERN-IHEP-JINR, 1-5 October 1990, Dubna, eds. S. Dubnicka, D. Ebert and A. Sazonov, World Scientific, Singapore, (1991), p. 288; Phys. Lett. B259 (1991) 144.

[4] E. Braaten, A. Pich and S. Narison, Nucl. Phys. B373 (1992) 581.

[5] A. C. Mattingly and P. M. Stevenson, preprint Rice Univ. DOE/ER/05096-50 (1992).

[6] F. Le Diberder and A. Pich, Phys. Lett. B286 (1992) 147.

[7] S. A. Larin, F. V. Tkachov and J. A. M. Vermaseren, Phys. Rev. Lett. 66 (1991) 862.

[8] S. A. Larin and J. A. M. Vermaseren, Phys. Lett. B259 (1991) 345.

[9] K. G. Chetyrkin, S. G. Gorishny, S. A. Larin and F. V. Tkachov, Phys. Lett. 137B (1984) 230.

[10] S. G. Gorishny and S. A. Larin, Phys. Lett. B172 (1986) 109.

[11] CCFR Collab., M. Shaevitz, Talk at the XXVII Recontre de Moriond on "QCD and High Energy Hadronic Interactions", 22-28 March 1992, Les Arcs; to appear in the Proceedings, ed. J. Tran Thanh Van;

W. C. Leung et al., Nevis Preprint 1460, June 1992.

[12] D. Duke and J. Owens, Phys. Rev. D30 (1984) 49.

[13] M. Diemoz, F. Ferroni, E. Longo and G. Martinelli, Z. Phys. C39 (1988) 21.

[14] J. Morfin and W. K. Tung, Z. Phys. C52 (1991) 13.

[15] R. L. Jaffe and M. Soldate, Phys. Rev. D26 (1982) 49;

E. V. Shuryak and A. I. Vainshtein, Nucl. Phys. B199 (1982) 951;

R. K. Ellis, W. Furmanski and R. Petronzio, Nucl. Phys. B207 (1982) 1; B212 (1983) 29.

[16] V. M. Braun and A. V. Kolesnichenko, Nucl. Phys. B283 (1987) 723. 
[17] V. I. Zakharov, Max-Planck-Institut preprint MPI-Ph/92-33 (1992).

L. S. Brown, L. G. Yaffe and Chengxing Zhai, Univ. of Washington preprint UW/PT92-07 (1992).

[18] S. Fajfer and R. J. Oakes, Phys. Lett. 163B (1985) 385.

[19] P. M. Stevenson, Phys. Rev. D23 (1981) 2916.

[20] O. V. Tarasov, A. A. Vladimirov and A. Yu. Zharkov, Phys. Lett. 93B (1980) 429.

[21] A. L. Kataev, preprint CERN-TH.6485/92 (1992); in Proc. XXVII Recontre de Moriond on "QCD and High Energy Hadronic Interactions" 22-28 March 1992, Les Arcs, ed. J. Tran Thanh Van (to appear).

[22] G. Grunberg, Phys. Lett. B221 (1980) 70; preprint Ecole Polytechnique A510.078 (1982) (unpublished); Phys. Rev. D29 (1984) 2315.

[23] A. Dhar and V. Gupta, Phys. Rev. D29 (1984) 2822.

[24] W. Marciano, Phys. Rev. D29 (1984) 580.

[25] V. I. Vovk, Z. Phys. C47 (1990) 57.

[26] A. V. Kotikov, G. Parente and J. Sanchez Guillen, preprint Univ. of Bern BUTP-91/40 (1991).

[27] M. Virchaux and A. Milsztajn, Phys. Lett. B274 (1992) 221.

[28] C. Sachrajda, Talk at the "QCD-20 Years Later" Conf. 9-13 June 1992, Aachen; to appear in the Proceedings.

[29] S. Bethke and S. Catani, preprint CERN-TH.6484/92 (1992); in Proc. XXVII Recontre de Moriond "QCD and High Energy Hadronic Interactions" 22-28 March 1992, Les Arcs, ed. J. Tran Thanh Van (to appear).

[30] V. Branchina, M. Consoli, R. Fiore and D. Zappala, Phys. Rev. D46 (1992) 75.

[31] I. I. Balitsky, V. M. Braun and A. V. Kolesnichenko, Phys. Lett. B242 (1990) 245.

[32] M.Anselmino, B.L.Ioffe and E.Leader, Yad.Fyz. 49 (1989) 214. 


\section{Figure captions}

Fig.1: The fractions $w^{i}, i=1,2,3$ as the functions of $Q^{2}$ for three widely used sets of quark distributions functions.

Fig.2: The dependence of $\Delta(\rho)$ from $n_{f}$ for the PMS/EC approaches and the $\overline{\mathrm{MS}} \mathrm{RS}$ (denoted in this and allthe following figures by MSb), at the NLO and the NNLO.

Fig.3: The RS dependence of $\Delta(\rho)$ for $n_{f}=3,4,5$ at the NLO and the NNLO.

Fig.4: The comparison between the NLO and the NNLO results for $\Delta(\rho)$ in the cases of $n_{f}=3,4,5$.

Note: In the process of the study of Fig.1-Fig.4 the readers should cnange MS to $\overline{M S}$ everywhere. This was not done in view of the technical problems in dealing with the corresponding PS-file. 


\begin{tabular}{|c|c|c|c|c|c|c|c|c|}
\hline & $\mathrm{RS}$ & $n_{f}$ & $r_{0}^{(i)}$ & $r_{1}^{(i)}$ & $r_{2}^{(i)}$ & $r_{3}^{(i)}$ & $r_{4}^{(i)}$ & $r_{5}^{(i)}$ \\
\hline \multirow{9}{*}{$\mathrm{N}$} & \multirow{3}{*}{ PMS } & 3 & 0.008137 & 0.645500 & 0.133457 & -0.413737 & -3.100763 & 7.573763 \\
\hline & & 4 & 0.008448 & 0.653533 & 0.076082 & -1.053512 & -3.038260 & 7.176102 \\
\hline & & 5 & 0.004056 & 0.814661 & -1.174005 & 0.267708 & 2.229508 & -2.753092 \\
\hline & \multirow{3}{*}{$\mathrm{ECH}$} & 3 & 0.007837 & 0.653591 & 0.081509 & -0.416852 & -2.659066 & 7.374106 \\
\hline & & 4 & 0.007270 & 0.691163 & -0.264749 & -0.428416 & -1.482951 & 3.615289 \\
\hline & & 5 & 0.003300 & 0.834586 & -1.291855 & 0.353730 & 2.839872 & -3.593072 \\
\hline & \multirow{3}{*}{$\overline{\mathrm{MS}}$} & 3 & 0.004927 & 0.760169 & -0.963719 & 0.123290 & 2.019464 & -2.445137 \\
\hline & & 4 & 0.008325 & 0.660719 & -0.062042 & -1.260045 & -2.644704 & 7.022455 \\
\hline & & 5 & 0.008725 & 0.667920 & -0.016647 & -1.436806 & -2.770146 & 7.534455 \\
\hline \multirow{9}{*}{$\mathrm{L}$} & \multirow{3}{*}{ PMS } & 3 & 0.004645 & 0.761183 & -0.904944 & 0.435364 & 2.299329 & -3.332708 \\
\hline & & 4 & 0.008607 & 0.648385 & 0.155498 & -1.095457 & -3.068545 & 7.277303 \\
\hline & & 5 & 0.006449 & 0.732606 & -0.245552 & -0.864299 & 0.201203 & 2.163065 \\
\hline & \multirow{3}{*}{$\mathrm{ECH}$} & 3 & 0.004908 & 0.754859 & -0.903029 & 0.442944 & 1.925275 & -2.829597 \\
\hline & & 4 & 0.004350 & 0.782570 & -0.926641 & 0.446002 & 2.117838 & -3.104819 \\
\hline & & 5 & 0.008235 & 0.675962 & 0.148810 & -1.066902 & -3.437890 & 7.772996 \\
\hline & \multirow{3}{*}{$\overline{\mathrm{MS}}$} & 3 & 0.005703 & 0.745372 & -1.420683 & 0.564036 & 3.195462 & -4.264516 \\
\hline & & 4 & 0.002404 & 0.851741 & -2.019862 & 2.597744 & -0.376554 & -1.574096 \\
\hline & & 5 & 0.008854 & 0.668406 & -0.247080 & -1.211435 & -2.402028 & 6.958375 \\
\hline
\end{tabular}

Table 1: The values of the coefficients $r_{j}^{(i)}$ for the $i-t h$ option where the option is defined by the combination of the order, RS and $n_{f}$. 


\begin{tabular}{|c|c|c|c|c|c|c|c|}
\hline & $\mathrm{RS}$ & $n_{f}$ & $\rho$ & $\Lambda \frac{(4)}{\mathrm{MS}}$ & $\sum_{i=3}^{5} w^{i} \Lambda_{\frac{(i)}{\mathrm{MS}}}$ & $\Delta^{(i)}$ & $\sum_{3}^{5} w^{(i)} \Delta^{(i)}$ \\
\hline \multirow{6}{*}{$\begin{array}{l}\mathrm{N} \\
\mathrm{N}\end{array}$} & \multirow{3}{*}{ PMS } & 3 & 4.93 & 191 & \multirow{3}{*}{$250 \pm 16(67)$} & 0.170 & \multirow{4}{*}{0.139} \\
\hline & & 4 & 4.65 & 233 & & 0.142 & \\
\hline & & 5 & 4.40 & 321 & & 0.117 & \\
\hline & \multirow{3}{*}{$\overline{\mathrm{MS}}$} & 3 & 4.25 & 226 & \multirow{3}{*}{$267 \pm 18(79)$} & 0.151 & \\
\hline & & 4 & 4.35 & 250 & & 0.140 & \multirow[t]{2}{*}{0.137} \\
\hline & & 5 & 4.45 & 317 & & 0.121 & \\
\hline \multirow{6}{*}{$\begin{array}{l}\mathrm{N} \\
\mathrm{L}\end{array}$} & \multirow{3}{*}{ PMS } & 3 & 4.50 & 212 & \multirow{3}{*}{$240 \pm 14(60)$} & 0.147 & \multirow{4}{*}{0.136} \\
\hline & & 4 & 4.70 & 230 & & 0.140 & \\
\hline & & 5 & 5.00 & 278 & & 0.122 & \\
\hline & \multirow{3}{*}{$\overline{\mathrm{MS}}$} & 3 & 3.10 & 300 & \multirow{3}{*}{$318 \pm 23(99)$} & 0.139 & \\
\hline & & 4 & 3.50 & 307 & & 0.138 & \multirow[t]{2}{*}{0.135} \\
\hline & & 5 & 4.00 & 352 & & 0.127 & \\
\hline
\end{tabular}

Table 2: Results of the procedure described in the text for the case with twist-four contribution given by the central value of the estimate (5). The values of the $\Lambda$-parameter are in $\mathrm{MeV}$ and the first (second) error corresponds to the statistical (systematical) experimental errors.

\begin{tabular}{|l|l|l|l|l|l|}
\hline & RS & HT=0 & HT=0.016 & HT=0.032 & HT=0.048 \\
\hline \multirow{2}{*}{ NNL } & PMS & $333 \pm 15(65)$ & $294 \pm 15(65)$ & $250 \pm 16(67)$ & $204 \pm 16(70)$ \\
\cline { 2 - 6 } & $\overline{M S}$ & $360 \pm 19(83)$ & $313 \pm 19(81)$ & $267 \pm 18(79)$ & $215 \pm 17(74)$ \\
\hline \multirow{2}{*}{ NL } & PMS & $319 \pm 13(55)$ & $281 \pm 13(55)$ & $240 \pm 14(60)$ & $201 \pm 14(60)$ \\
\cline { 2 - 6 } & $\overline{M S}$ & $435 \pm 20(87)$ & $378 \pm 21(90)$ & $318 \pm 23(99)$ & $254 \pm 24(105)$ \\
\hline
\end{tabular}

Table 3: The values of $\Lambda \frac{(4)}{\mathrm{MS}}$ (in $\mathrm{MeV}$ ) as extracted from data of the CCFR Collaboration under different assumptions of the higher twist (HT) contribution. The errors are the same as in the Table 2 . 